\title{
Crystal Structure of Human Peroxiredoxin 5, a Novel Type of Mammalian Peroxiredoxin at $1.5 \AA$ Resolution
}

\author{
Jean-Paul Declercq ${ }^{1}$, Christine Evrard ${ }^{1}$, André Clippe ${ }^{2,3}$ Delphine Vander Stricht $^{2}$, Alfred Bernard ${ }^{3}$ and Bernard \\ Knoops $^{2}$ \\ ${ }^{1}$ Unit of Structural Chemistry (CSTR), Université Catholique de Louvain, 1 place Louis Pasteur, B-1348, Louvain-la-Neuve, Belgium \\ ${ }^{2}$ Laboratory of Cell Biology Université Catholique de Louvain, 5 place Croix du Sud B-1348, Louvain-la-Neuve Belgium \\ ${ }^{3}$ Unit of Industrial Toxicology and Occupational Medicine Université Catholique de Louvain, 30 clos Chapelle-aux-Champs, B-1200, \\ Brussels Belgium
}

\begin{abstract}
The peroxiredoxins define an emerging family of peroxidases able to reduce hydrogen peroxide and alkyl hydroperoxides with the use of reducing equivalents derived from thiol-containing donor molecules such as thioredoxin, glutathione, trypanothione and AhpF. Peroxiredoxins have been identified in prokaryotes as well as in eukaryotes. Peroxiredoxin 5 (PRDX5) is a novel type of mammalian thioredoxin peroxidase widely expressed in tissues and located cellularly to mitochondria, peroxisomes and cytosol. Functionally, PRDX5 has been implicated in antioxidant protective mechanisms as well as in signal transduction in cells. We report here the 1.5 $\AA$ resolution crystal structure of human PRDX5 in its reduced form. The crystal structure reveals that PRDX5 presents a thioredoxin-like domain. Interestingly, the crystal structure shows also that PRDX5 does not form a dimer like other mammalian members of the peroxiredoxin family. In the reduced form of PRDX5, Cys47 and Cys151 are distant of $13.8 \AA \AA$ although these two cysteine residues are thought to be involved in peroxide reductase activity by forming an intramolecular disulfide intermediate in the oxidized enzyme. These data suggest that the enzyme would necessitate a conformational change to form a disulfide bond between catalytic Cys47 and Cys151 upon oxidation according to proposed peroxide reduction mechanisms. Moreover, the presence of a benzoate ion, a hydroxyl radical scavenger, was noted close to the active-site pocket. The possible role of benzoate in the antioxidant activity of PRDX5 is discussed.
\end{abstract}

Keywords: antioxidant enzyme; peroxiredoxin; thioredoxin fold; thioredoxin peroxidase; crystal structure

Abbreviations used: PRDX, peroxiredoxin; ROS, reactive oxygen species; PrxV, peroxiredoxin V; AOEB166, antioxidant enzyme B166; PMP20, peroxisomal membrane protein 20; TNF $\alpha$, tumor necrosis factor alpha; AhpC, alkyl hydroperoxide reductase subunit C; PCR, polymerase chain reaction; PBS, phosphate-buffered saline; MAD, multiwavelength anomalous dispersion; GC, gas chromatography; EI, electron impact; MS, mass spectrometry.

\section{INTRODUCTION}

Peroxiredoxins are a family of antioxidant enzymes evolutionarily conserved that so far include more than 40 members identified in a variety of organisms from bacteria, yeasts and protozoa to plants and vertebrates. ${ }^{1}$ Peroxiredoxins cooperate with other antioxidant enzymes and non-enzymatic antioxidants to regulate the levels of reactive oxygen species (ROS) in cells and tissues, and to protect them against oxidative attacks. Indeed, ROS and their toxic by-products may react with lipids, proteins and nucleic acids, leading to cell damage and necrotic or apoptotic cell death. On the other hand, recent studies have implicated ROS in normal physiological signalling by growth factors and cytokines suggesting that enzymes that regulate ROS levels in cells may also be involved in the control of signal transduction. ${ }^{2,3}$

Peroxiredoxin 5 (PRDX5), also known as PrxV/ AOEB166/PMP20/ACR1, is a novel thioredoxin peroxidase widely expressed in mammalian tissues. ${ }^{2,4-6}$ PRDX5 may be addressed intracellu-larly to mitochondria, peroxisomes and the cytosol, suggesting that this peroxiredoxin may have an important role as antioxidant in organelles that are major sources of ROS, namely mitochondria and peroxisomes, and in the control of signal transduction due to its localization in the cytosol. ${ }^{2,3,5,6}$ Moreover, the physiological importance of PRDX5 has recently been emphasized by its ability to prevent $\mathrm{p} 53$-induced apoptosis and to inhibit intracellular hydrogen peroxide accumulation by TNF $\alpha .{ }^{2,3}$ PRDX5 is able to reduce hydrogen peroxide and alkyl hydroperoxides. ${ }^{2,6} \mathrm{~A}$ peroxinitrite reductase activity of AhpC, the bacterial peroxire-doxin orthologue of human PRDX5, has recently been reported, suggesting a similar activity for its human counterpart. ${ }^{7}$ 
All peroxiredoxins contain a conserved Cys residue in the $\mathrm{N}$-terminal portion of the protein, which is oxidized by peroxides to sulfenic acid (Cys-SOH). In PRDX1 to PRDX4, which represent the so-called 2-Cys mammalian subgroup, this sulfenic acid reacts with a conserved C-terminal Cys-SH of another subunit to form an intermolecular disulfide. This disulfide is then reduced by thioredoxin. In contrast, PRDX5 forms a reaction intermediate distinct from the 2-Cys subgroup. Indeed, the $\mathrm{N}$-terminal sulfenic acid (Cys47) reacts with a Cterminal Cys-SH (Cys151) of the same molecule and thereby forms an intramolecular disulfide intermediate that can be reduced by thioredoxin. ${ }^{2}$ For PRDX6, the only known mammalian member of the 1-Cys subgroup, the mechanism by which the sulfenic acid is reduced is still to be elucidated.

The recent X-ray structure of PRDX1, PRDX2 and PRDX6 has shown that peroxiredoxins are novel members of the thioredoxin fold superfam-ily, which includes thioredoxins, glutaredoxins, glutathione-S-transferases, protein disulfide bond isomerases and glutathione peroxidases. ${ }^{8-11}$ Here, we describe the 1.5 Á resolution crystal structure of Escherichia coli recombinant human PRDX5 in reduced form. Our structural data reveal that PRDX5 represents a prototype of a novel type of mammalian peroxiredoxin. Some particular features of its catalytic site revealed by X-ray crystallography suggest that PRDX5 could present a broader activity against ROS compared to other peroxiredoxins or antioxidant enzymes that are addressed to the same subcellular compartments.

\section{RESULTS}

\section{Overall structure}

PRDX5 is characterized by a molecular mass of $17 \mathrm{kDa}$ and exists as a single-domain monomelic protein of

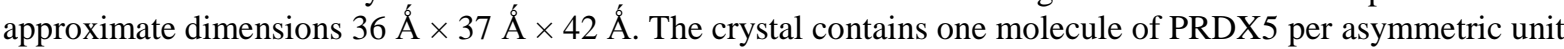
and presents a relatively loose packing with a solvent content about $65 \%(\mathrm{v} / \mathrm{v})$ resulting in large channels of solvent surrounding the molecules. The crystal structure of PRDX5 is illustrated in Figure 1. It consists of 161 residues that fold into six $\alpha$-helices $(\alpha 1-\alpha 6)$ and seven $\beta$-strands $(\beta 1-\beta 7)$. No interpretable electron density is present for the N-terminal $6 \times$ Histail and its short linker. The secondary structural elements comprise a typical thioredoxin fold, which is formed by a central four-stranded $\beta$-sheet $(\beta 3, \beta 4, \beta 6, \beta 7)$ and three flanking $\alpha$-helices $(\alpha 2, \alpha 4, a 6)$. In addition to the thioredoxin fold, PRDX5 presents at its $\mathrm{N}$-terminal end an extra two-stranded $\beta$ sheet $(\beta 1, \beta 2)$ immediately followed by a short $\alpha$-helix $(\alpha 1)$. Another $\alpha$-helix $(\alpha 3)$ and a $\beta$-strand $(\beta 5)$ which forms a fifth strand in the thioredoxin fold $\beta$-sheet, running parallel with the $\beta 4$ strand, are inserted into the sequence between $\beta 4$ and $\alpha 4$. Finally, an additional $\alpha$-helix ( $\alpha 5)$ is incorporated in the loop connecting $\alpha 4$ and $\beta 6$.

\section{Scheme 1.}

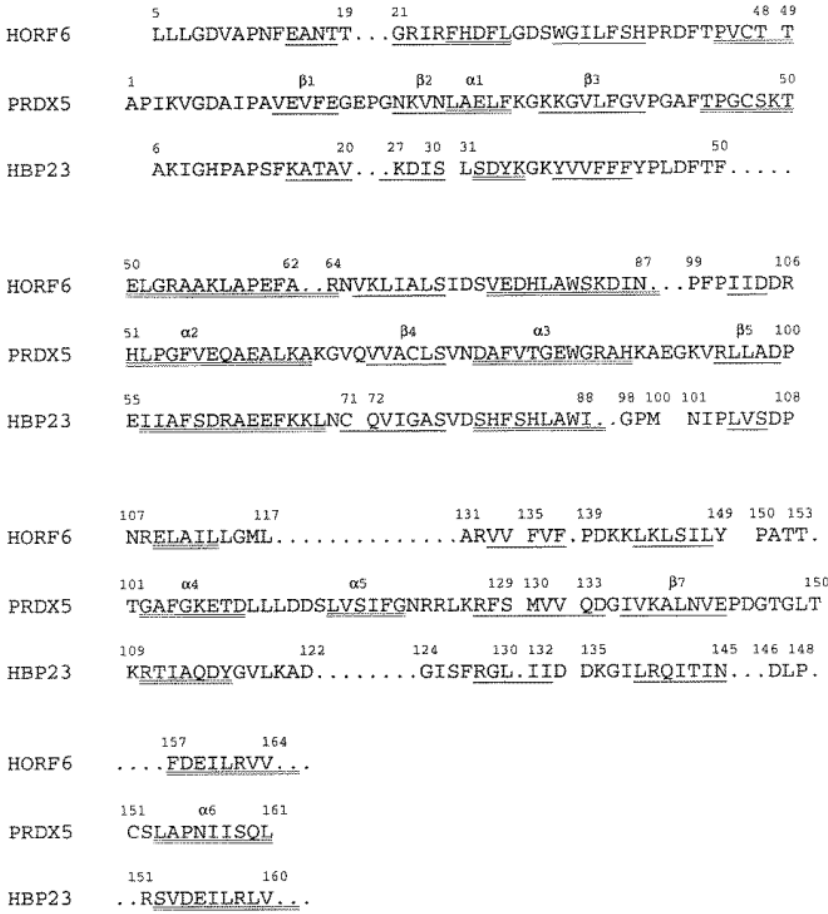




\section{DISCUSSION}

\section{Structural comparison with human PRDX6 (hORF6) and rat PRDX1 (HBP23)}

For a structural comparison with other known peroxiredoxins, we have selected hORF6 ${ }^{9}$ and $\mathrm{HBP} 23^{10}$ as representatives of the 1-Cys and 2-Cys peroxiredoxin subgroups, respectively. ${ }^{2}$ The best structural alignments between the $C^{\alpha}$ atoms of PRDX5 and of these two molecules was searched using the program LSQMAN ${ }^{12}$ and refined with lsq_imp in O. ${ }^{13}$ This allowed us to align 131 and $136 \mathrm{C}^{\alpha}$ atoms with a cut-off limit of $3.8 \AA$ and root-mean-square deviations of $1.73 \AA$ and $1.88 \AA$ for hORF6 and HBP23, respectively. The superposition of the $\mathrm{C}^{\alpha}$ atoms presented in Figure 2 shows that in PRDX5, only the N-terminal domains of hORF6 and HBP23 are present, while the C-terminal domain is completely absent, in agreement with previous sequence alignments. ${ }^{6}$ In Scheme 1, the complete sequence of PRDX5 is structurally aligned with parts of the two other sequences. The secondary structural elements of PRDX5 are labelled and show that good structural comparisons occur for the elements taking part in the thioredoxin fold $(\beta 3, \alpha 2, \beta 4, \alpha 4, \beta 6, \beta 7, \alpha 6)$. Most of the remaining secondary structures $(\beta 1, \beta 2, \alpha 1, \alpha 3, \beta 5)$ are also conserved in the three structures, with the exception of helix $\alpha 5$, which is present only in PRDX5. The N-terminal part of helix $\alpha 2$ is well superposed in PRDX5 and hORF6 with a good coincidence of the catalytic residues Cys47 $\left(\mathrm{C}^{\alpha}-\mathrm{C}^{\alpha} 0.78 \AA\right.$ ), while in HBP23, this part of the helix is unwound and contains the corresponding catalytic residue Cys52. The kink of helix $\alpha 2$, which occurs in the two other peroxiredoxin structures, is observed in PRDX5 at the level of Ala59 and is stabilized by a water molecule making hydrogen bonds with 057 and N61. Figure 2 shows that, in addition to helix $\alpha 5$, the largest discrepancies occur in the loops between $\beta 1-\beta 2, \alpha 3-\beta 5$ and $\beta 7-\alpha 6$ in which it is worth noting the presence of Cys 151 , an additional non-conserved cysteine residue implicated in the proposed peroxide reduction mechanism. ${ }^{2}$

Large differences are observed in the packing of PRDX5 in comparison with other peroxiredoxin structures. In hORF6 and HBP23, tightly associated dimers exist in the crystal by the formation of a hydrogen bonding network between two $\beta$-strands ( $\beta 7$ of each monomer) resulting in a ten-stranded $\beta$-sheet in the dimer. A similar situation is observed in TPx-B ${ }^{11}$ (PRDX2), a peroxiredoxin of the 2-Cys subgroup in which the $\beta$-sheets of two monomers combine to form a 14 -stranded $\beta$-sheet and, in this case, five dimers are associated to form a toroidshaped decamer. The dimeric form of HBP23 is reinforced by the presence of a disulfide bond between Cys52 of one molecule and Cys 173 of the other molecule. We do not observe this kind of dimer formation in PRDX5, where each molecule is surrounded by five other molecules without very tight association and with the formation of very large solvent channels. The side-chain of the additional cysteine residue, Cys 151, is well exposed in these solvent channels. The exact reason why PRDX5 does not form dimers remains an open question. It might be related to the peroxide reduction mechanism, since it was shown ${ }^{2}$ that PRDX5 forms an intramolecular disulfide as a reaction intermediate while in PRDX1 to PRDX4 (the 2-Cys mammalian subgroup) an intermolecular disulfide occurs ${ }^{10}$ that is favoured by the formation of the dimer. During the purification of PRDX5, we did not detect the existence of dimers, while they were observed in hORF $^{9}$ at concentrations higher than $1 \mathrm{mg} \mathrm{ml}^{-1}$. This observation suggests that the absence of dimer in the crystal structure of PRDX5 is independent of the different conditions of crystallization, since ammonium sulfate used for PRDX5 was not used for other PRDX structures. Furthermore, we do not observe any ordered sulfate ion in the region of the $\beta 7$ strand that could prevent the formation of a dimer. On the other hand, we have checked by model building the possible occurrence of steric factors that would prevent the formation of an interface between two monomers. The model of the dimer was built by superposing two PRDX5 molecules on the two molecules of the dimer of hORF6. At the C-terminal end of the $\beta 7$ strand, we observe in PRDX5 a kind of bump formed by residues Pro144-Asp145, which does not exist in hORF6 and HBP23 (see Scheme 1 and Figure 2) and which could obstruct the formation of a $\beta$-sheet between two symmetry-related $\beta 7$ strands, since we observe very close contacts $(<1.0 \AA$ Á) between Pro144 of one molecule and Ala139 of the other one. 
Figure 1. Overall view of the structure of PRDX5. (a) Topological diagram showing the arrangement of the secondary structural elements in PRDX5. The helices are represented as cylinders and the $\beta$-strands as arrows. The beginning and the end of the secondary structural elements are labelled. The three helices and the four $\beta$ strands belonging to the thioredoxin fold are colored green and red, respectively, while the remaining elements are colored yellow, $(b)$ and (c) Ribbon diagrams showing the overall organization of PRDX5, colored as in (a). The two orientations are nearly perpendicular, (c) being a top view of (b). The side-chains of the three Cys residues are represented as balls and sticks (Cys 72 is hidden in $(b))$. Parts $(b)$ and $(c)$ were prepared using MOLSCRIPT $^{26}$ and Raster $3 D .^{27}$
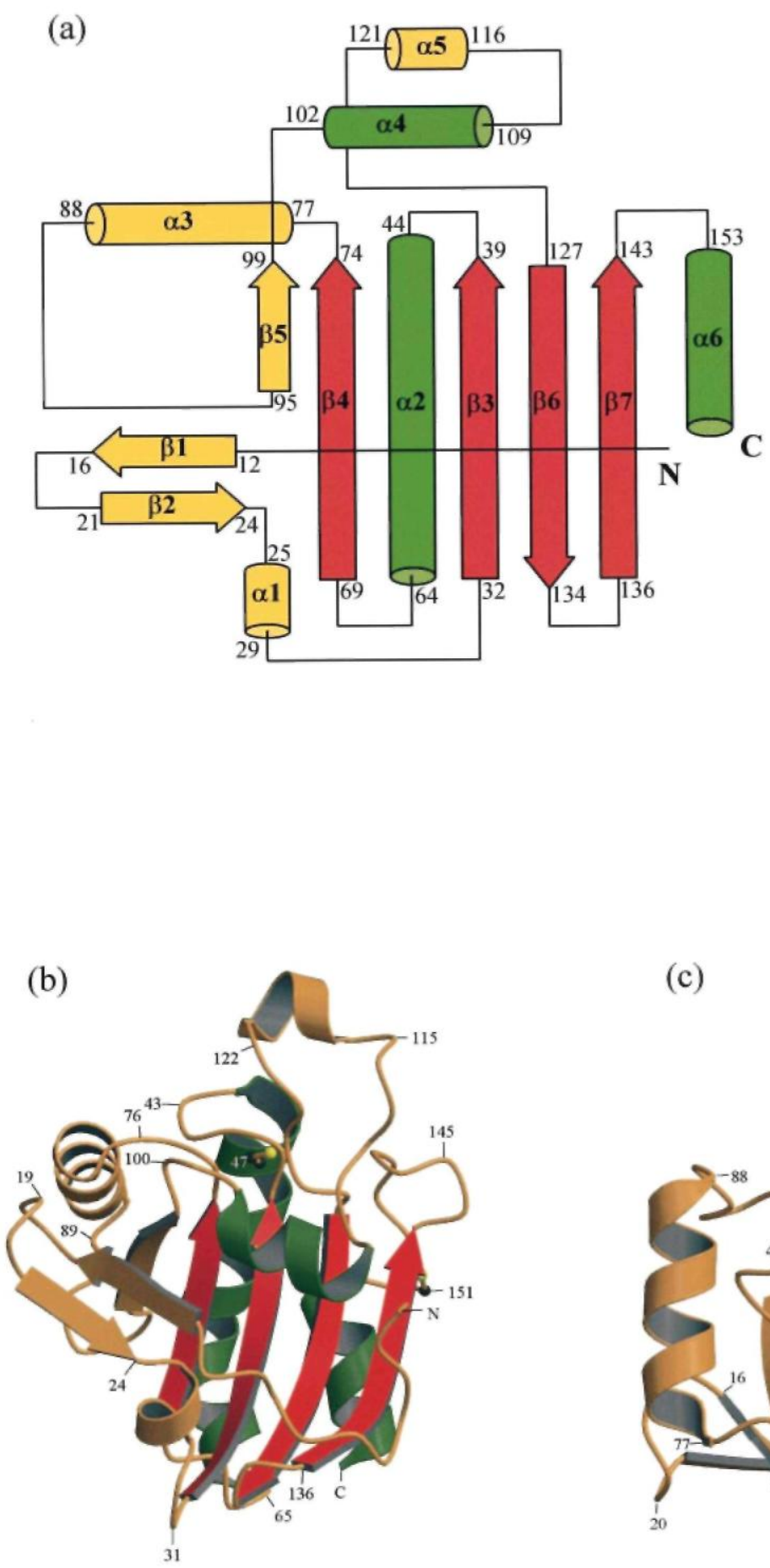

(c)

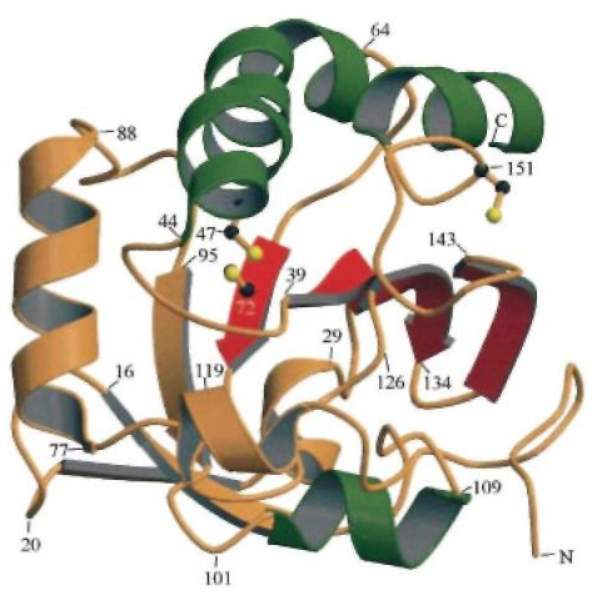


Figure 2. Structural comparisons with other peroxiredoxins. Stereoscopic view of a superposition between the $C^{\alpha}$ traces of PRDX5 (red) and (a) hORF6, (b) HBP23 (green). The orientation is the same as in Figure 1(b). The Figures were produced using $O .^{13}$
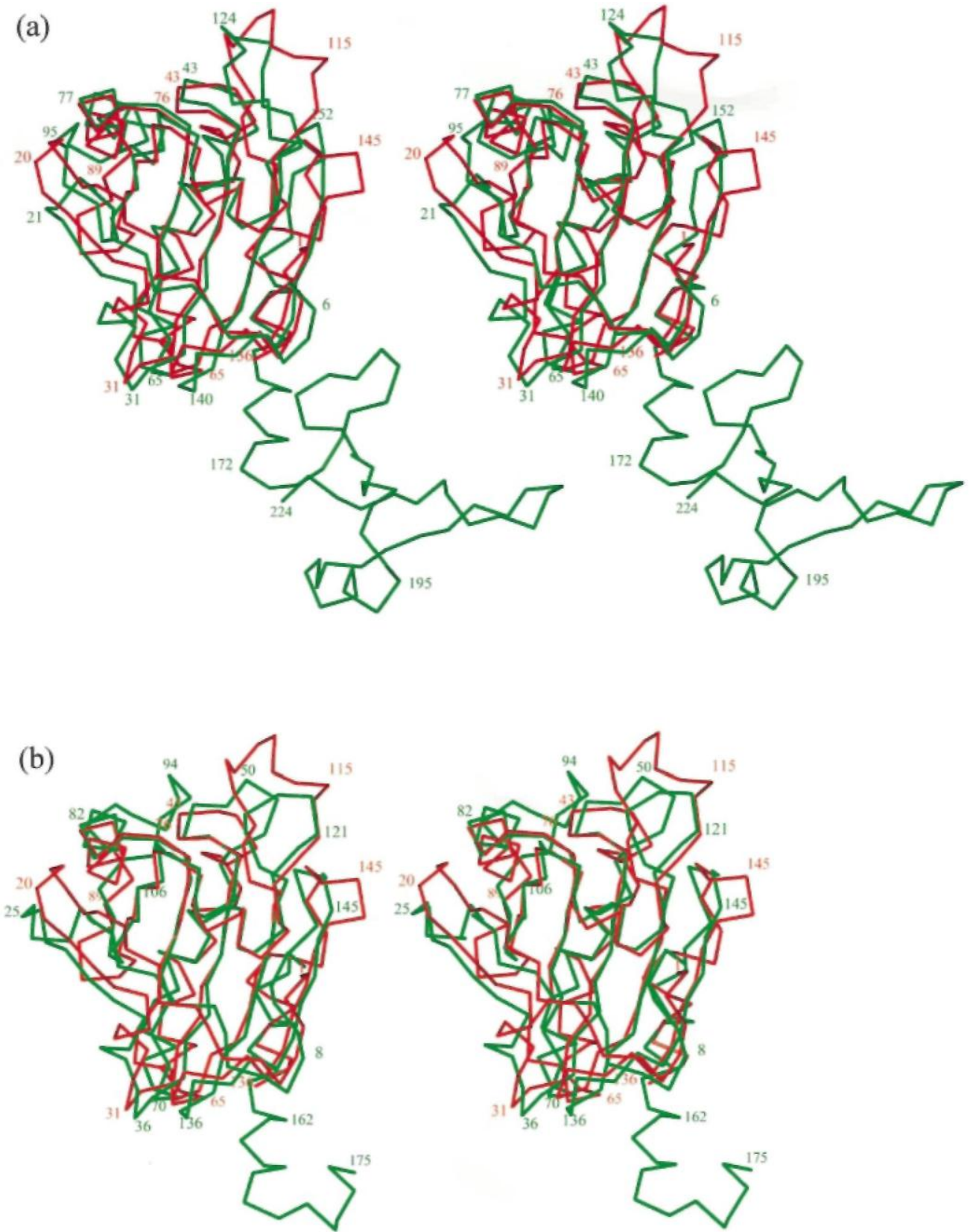

\section{Active site}

Presented as a novel member of the mammalian peroxiredoxin family, ${ }^{6}$ PRDX5 possesses one cysteine residue, Cys47, which is highly conserved in all the other peroxiredoxins (PRDX1 to 4 and PRDX6) ${ }^{14}$ and which has been implicated directly in catalysis of peroxides. PRDX5 contains no cysteine residue corresponding to the second conserved cysteine residue of the 2-Cys subgroup but presents two additional cysteine residues, lacking in the 1-Cys subgroup.

The conserved cysteine residue of PRDX5, Cys47, is located at the N-terminal part of the kinked helix $\alpha 2$, inside a small cavity. The environment of Cys47 is presented in Figure 3. In common with the 1-Cys peroxiredoxin hORF $6,{ }^{9}$ the active site consists of a positively charged pocket, largely exposed to solvent exterior. The catalytically active cysteine residues coincide in the two structures. 
However, in the recently solved crystal structure of the 2-Cys peroxiredoxin HPB23, ${ }^{10}$ the corresponding cysteine residue (Cys52) appears to be completely buried in the cavity and is significantly displaced from Cys 47 of PRDX5 $\left(C^{\alpha}-C^{\alpha}=6.8 \AA \AA\right)$. The crystal structure of hORF6 reveals a positively charged environment for the catalytically conserved cysteine residue (Cys47) in its oxidized form, cysteine-sulfenic acid (Cys-SOH). Good contacts are observed with His39 $\left(\mathrm{N}^{\delta 1}\right), \operatorname{Argl} 32\left(\mathrm{~N}^{\varepsilon 1}\right)$ and a possible magnesium ion. The authors suggest that these close interactions with Cys47 may play a role in lowering the $\mathrm{p} K_{\mathrm{a}}$ of the thiol by stabilizing its ionized state, increasing the reactivity. His39 is completely conserved in the 1-Cys subgroup but is replaced by Tyr or Trp in the 2-Cys subgroup. PRDX5 has a valine residue (Val39) in that position (distance between Cys47-S $\mathrm{S}^{\gamma}$ and Val39- $C^{\beta}=3.6 \AA$ ). Nevertheless, Arg132 is highly conserved in the 1-Cys and 2-Cys subgroups, and corresponds to $\operatorname{Arg} 128$ in the structure of HPB23. This arginine residue is found in the structure of PRDX5, namely $\operatorname{Arg} 127$, with its $\mathrm{N}^{\eta 1}$ interacting with the sulfur atom of Cys47 at a distance of $3.3 \AA$, and seems to be responsible for the positively charged active-site pocket (Figure 3(a)). In the crystal structure of hORF6, another arginine residue, $\operatorname{Arg} 155$, is found with its side-chain oriented in the direction of the active cysteine residue. In the structure comparison of hORF6-PRDX5, this residue is positioned not far from Cys 151 in PRDX5 (C ${ }^{\alpha}-C^{\alpha} 5.1$ $\AA$ ). Cys151 corresponds to the additional non-conserved cysteine residue implicated in the proposed mechanism of action of PRDX5. ${ }^{2}$ It is located in the loop connecting $\beta 7$ to $\alpha 6$, with its side-chain exposed to the solvent region. However, the sulfur atoms of Cys47 and Cys151 are positioned too far apart $\left(\mathrm{S}^{\gamma}-\mathrm{S}^{\gamma} 13.8 \AA \AA\right.$ ) to interact without large conformational changes. It is likely that such large conformational changes must occur in PRDX5 upon oxidation, since we observe that crystals of PRDX5 are destroyed immediately when soaked in the presence of $10^{-3} \mathrm{M}$ hydrogen peroxide, while crystals of the C47S mutant, in which the formation of the disulfide bond between residues 47 and 151 is no longer possible, survive many weeks under similar conditions. PRDX5 contains a third cysteine residue, Cys72, at the end of a $\beta$-strand, $\beta 4$. Interestingly, Cys 72 is located at the bottom of the active-site pocket, not far from the active-site, Cys47 $\left(S^{\gamma}-S^{\gamma} 7.4 \AA\right.$ ) though Seo et al. ${ }^{2}$ have shown that the mutation of Cys72 had no effect on activity. The corresponding residues in the crystal structures of hORF6 and HPB23 are Ala70 and Gly75, respectively. A threonine residue (Thr44) is present in the active-site cavity of PRDX5 with its oxygen atom $\mathrm{O}^{\gamma 1}$ clearly interacting with the sulfur atom of the catalytic cysteine residue (Cys47) at a distance of 3.0 Á. Additional electron density looking like a benzoate ion, whose identity was confirmed by mass spectrometry, is found close to the active-site pocket and restricts the access to the cavity. Good contacts are observed between the oxygen atom $\mathrm{O} 1$ of the benzoate ion and the sulfur atom of Cys47, which are $3.4 \AA$ apart. Moreover, one side of the active-site pocket contains several hydrophobic residues, including Leull6, Ile119 and Phe120, whose side-chains are located in the neighbourhood of the benzoate aromatic ring. Interestingly, these three hydrophobic residues are located in helix $\alpha 5$, which is not observed in other peroxiredoxins. The hydrophobic surrounding of the aromatic part of this benzoate ion is completed by the side-chain of Phe79 belonging to a symmetry-related molecule. In the absence of the benzoate ion, these hydrophobic residues would be exposed to solvent. Benzoic acid is known to be a specific scavenger of hydroxyl radical. ${ }^{15}$ Its localization close to the catalytic site suggests that it could be involved in the scavenging of hydroxyl radicals produced as reaction intermediates in the process of peroxide reduction. Alternatively, the implication of PRDX5 as specific hydroxyl radical scavenger via its benzoate ion can be postulated. 
Figure 3. View of the surrounding of the active-site pocket. (a) Molecular surface coloured according to the local electrostatic potential, ranging from blue (the most positive region) to red (the most negative). The positions of some residues discussed in the text are shown. (b) A diagram in which the side-chains of some important residues (see the text) are represented as balls and sticks, and labelled. The benzoate ion is also shown. The colors of the helices and $\beta$-strands are the same as in Figure 1. (c) Stereoscopic view oriented like (b) in which the electron density of a $\sigma-A^{28}$ map has been contoured at a level of $2 \sigma$ around the side-chains and the benzoate ion. Part (a) was produced using GRASP, ${ }^{29}(b)$ using MOLSCRIPT ${ }^{26}$ and Raster3D, ${ }^{27}$ (c) using $O .^{13}$
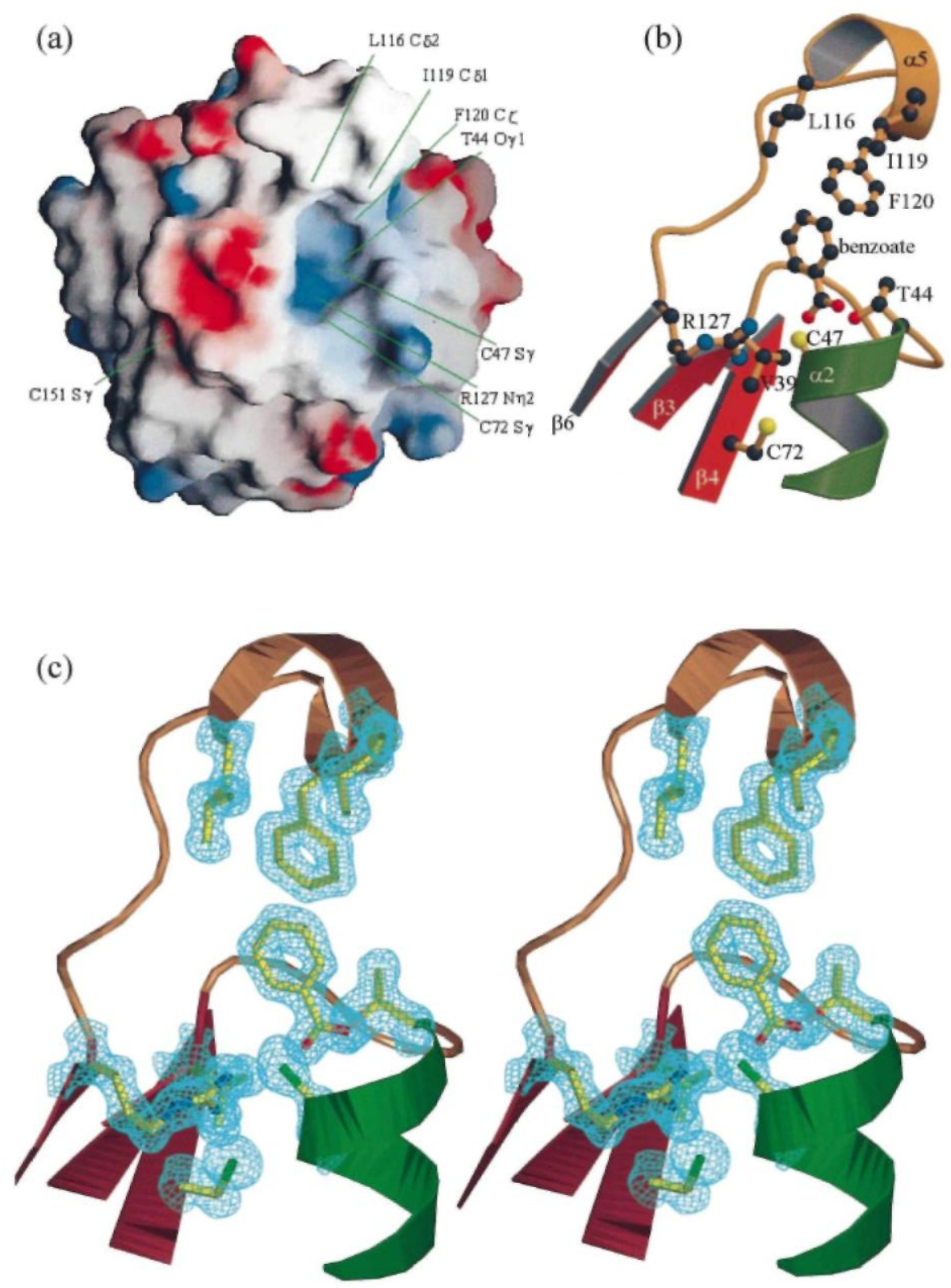


\section{MATERIALS AND METHODS}

\section{Bacterial expression of human PRDX5 and purification}

Human PRDX5 cDNA $^{6}$ was PCR amplified using forward primer 5'-GCTGCAGGATCCGCCCCAAT-

CAAGGTGGGAG-3' (BamHI site underlined) and reverse primer 5'-GGCCCAAAGCTTCAGAGCTGTGAGATGATA-3' (HindIII site underlined). The PCR product was digested with BamHI and HindIII, and ligated into the pQE-30 expression vector (Qiagen). The insert was sequenced and the N-terminal fusion with the hexahistidine $(6 \times$ His) tag was confirmed. The resulting vector was used to transform E. coli strain M15 (pRep4). E. coli were grown at $37^{\circ} \mathrm{C}$ in LB medium containing $1 \mathrm{mM}$ IPTG. Pelleted cells were lysed in $10 \mathrm{mM}$ imidazole, $50 \mathrm{mM}$ phosphate, $300 \mathrm{mM} \mathrm{NaCl}(\mathrm{pH} 8)$ by soni-cation and clarified by centrifugation. The supernatant containing $6 \times$ His-tagged PRDX5 was loaded onto a Ni ${ }^{2+}$-NTA column (Qiagen). The column was washed and the protein was eluted with $50 \mathrm{mM}$ phosphate, $300 \mathrm{mM} \mathrm{NaCl}, 250 \mathrm{mM}$ imidazole ( $\mathrm{pH}$ 8). Eluted protein was then dialysed against PBS ( $\mathrm{pH} 7.2$ ) and stored at $-20^{\circ} \mathrm{C}$ before use for crystallization.

\section{Crystallization}

The crystals were grown by hanging drop vapour diffusion at $18{ }^{\circ} \mathrm{C}$ by mixing $2 \mu 1$ of the protein solution (10 $\mathrm{mg} \mathrm{ml}^{-1}$ ) with $2 \mu \mathrm{l}$ of the well solution (volume $500 \mu \mathrm{l}$ ) composed of $1.6 \mathrm{M}$ ammonium sulfate, $0.1 \mathrm{M}$ sodium citrate buffer ( $\mathrm{pH}$ 5.3), $0.2 \mathrm{M}$ potassium sodium tartrate, $10^{-3} \mathrm{M}$ 1,4-dithio-dl-threitol, $0.02 \%$ (w/v) sodium azide. Crystals of octahedral shape appeared after four or five days with typical dimensions $0.2-0.3 \mathrm{~mm}$. The crystals were very unstable and they disappeared spontaneously about one week later.

Table 1. Data collection, phasing and refinement statistics

\begin{tabular}{|c|c|c|c|c|}
\hline Data set & High-resolution & Inflexion point & Peak & Remote \\
\hline Wavelength $(\AA ̊)$ & 1.1 & 0.9175 & 0.9169 & 0.855 \\
\hline Resolution range $(\AA)$ & $16-1.5$ & $16-1.7$ & $15-1.9$ & $15-1.7$ \\
\hline \multicolumn{5}{|l|}{ Reflections } \\
\hline Total & 384,543 & 239,367 & 168,023 & 287,845 \\
\hline Unique & 45,181 & 31,215 & 22,522 & 31,228 \\
\hline Completeness $(\%)$ & 100.0 & 100.0 & 100.0 & 99.9 \\
\hline$R_{\text {merge }}$ & 0.051 & 0.067 & 0.090 & 0.081 \\
\hline Cullis $R(1.9 \AA)$ (acentric/centric/anomalous) & $0.61 / 0.66 / 0.94$ & $-/-/ 0.87$ & $0.97 / 0.98 / 0.81$ & $0.62 / 0.67 / 0.78$ \\
\hline Phasing power (1.9 $\AA$ ) (acentric/centric) & $1.98 / 1.18$ & & $0.27 / 0.19$ & $1.92 / 1.15$ \\
\hline FOM (mlphare/dm) $(1.9 \AA / 1.7 \AA)$ & $0.67 / 0.85$ & & & \\
\hline R-factor $\left(R_{\text {free }}\right)(1.5 \AA)$ & $0.133(0.165)$ & & & \\
\hline \multicolumn{5}{|l|}{ RMS deviation from ideality } \\
\hline Bonds $(\AA)$ & 0.015 & & & \\
\hline Angles $\left({ }^{\circ}\right)$ & 2.2 & & & \\
\hline \multicolumn{5}{|l|}{ Average B-factors $\left(\AA^{2}\right)$} \\
\hline Main-chain & 13.5 & & & \\
\hline Side-chain & 22.8 & & & \\
\hline Solvent & 31.5 & & & \\
\hline Bromide ions & 24.4 & & & \\
\hline Benzoate & 20.1 & & & \\
\hline
\end{tabular}




\section{Data collection, structure determination and refinement}

Since our aim was to determine the phases by the multiwavelength anomalous dispersion (MAD) method after derivatization by short cryo-soaking with halides, ${ }^{16}$ crystals were transferred to solutions containing the compounds of the crystallization buffer, $1.0 \mathrm{M}$ sodium bromide and $20 \%$ (v/v) glycerol for about 30 seconds before flash-cooling at $100 \mathrm{~K}$. During these cryogenic freezing experiments, it clearly appeared that this crystal form did not tolerate glycerol alone, in the absence of sodium bromide. All diffraction data (four wavelengths) were collected from the same crystal at $100 \mathrm{~K}$ using the synchrotron X31 beam line, at EMBL c/o DESY (Hamburg) and a MAR345 imaging plate detector. One wavelength (1.1 $\AA$ ) was chosen to achieve the highest resolution, while the three other wavelengths were selected according to the $\mathrm{Br} / \mathrm{K}$ edge as determined by an $\mathrm{X}$ ray fluorescence scan: inflexion point, peak and remote on the high-energy side. All data were collected in a single pass using a rotation of $0.7^{\circ}$ or $1^{\circ}$. Statistics of data collection and processing are given in Table 1 . The crystals are tetragonal, space group $P 4_{1} 22_{1} 2$, with $a=66.61 \AA ̊, c=123.33 \AA$ and one protein molecule in the asymmetric unit. All the measurements were indexed and integrated using the program DENZO ${ }^{17}$ and merged with the program SCALEPACK: ${ }^{17} 5 \%$ of the reflections were flagged for use in $R_{\text {free }}$ calculations. ${ }^{18}$

The structure was determined by the MAD method using the anomalous signal of bromine atoms and considering as "native" the data collected at the inflexion point. The program SHELXS- $97^{19}$ allowed us to identify the same two sites from the Patterson functions based on anomalous differences at the peak wavelength and on dispersive differences $\left(\lambda_{\text {remote }}-\lambda_{\text {inflexion }}\right)$. Three additional sites were found from difference Fourier synthesis and were checked for consistency with the dispersive difference Patterson. Considering the contacts $(<3.5 \AA$ Á) of the bromide ions, we observe that all of them are in hydrogen bond interaction with one or more solvent molecules. In addition, three of these anions are bound to the main-chain N atoms of Glu91, Asp113 and Leul49, the last one being in contact also with the side-chain atom $\mathrm{N}^{\varepsilon 2}$ of Gln133 belonging to a symmetryrelated molecule. One of the bromide anions is bound to $\mathrm{O}^{\gamma}$ of Ser118 and to two $\mathrm{N}^{\zeta}$ atoms of Lys residues (63 and 93) in a symmetry-related molecule. MLPHARE ${ }^{20,21}$ was used for refining the heavy-atom positions and for computing the initial phases at $1.9 \AA$ resolution. These phases were then applied to the high-resolution data set and further improved by density modifications in $\mathrm{DM}^{20,22}$ using solvent flattening, histogram mapping and multiresolution modification, and extended to $1.7 \mathrm{~A}$. The program ARP/WARP ${ }^{23}$ in mode warpNtrace and side_dock succeeded in building 158 of the 161 residues. Only a few manual adjustments were necessary using the molecular graphics program O. ${ }^{13}$ The N-terminal His tail and its short linker were never observed, suggesting a complete disorder of these residues. The refinement at $1.5 \AA$ was performed with SHELXL-97. ${ }^{24}$ Alternate conformations were observed for the side-chains of residues Asn21 and Lys63. Five bromide ions and 221 ordered solvent molecules were incorporated and a bulk solvent correction was applied. A small molecule looking like a benzoate ion was observed at the entrance of the cleft containing the active site (Cys47) and introduced in the refinement. The presence of a benzoate ion in the protein solution before the addition of the crystallization buffer was confirmed by mass spectrometry (GC-electron impact (EI), mass spectrometry (MS) and MS-MS). Benzoate was not used in the production or purification steps. This model resulted in an $R$-value of $0.192\left(R_{\text {free }}=0.215\right)$ for all available data. A significant improvement was reached by applying restrained anisotropic temperature factors and yielded an R-value of $0.143\left(\mathrm{R}_{\text {free }}=0.178\right)$. After minor manual adjustments and the introduction of hydrogen atoms in riding positions, the $R$-value was $0.133\left(R_{\text {free }}=0.165\right)$. During the very last refinement cycles, the $R_{\text {free }}$ flagged reflections were included and the final R-value for all available reflections is 0.133 (no cut-off). A Ramachandran plot computed with the program PROCHECK ${ }^{25}$ shows that $87.8 \%$ of the residues are in the most-favoured regions and that there are no residues in disallowed regions.

\section{Protein Data Bank accession numbers}

Final coordinates and structure factors have been deposited with the Protein Data Bank under accession numbers 1HD2 and R1HD2SF, respectively.

\section{Acknowledgments}

This work was supported by grants from the Fonds National de la Recherche Scientifique (Belgium) and from the European Union key action "Environment and Health" (CT 99 QLK4-1308). A.B. is Research Director of the Fonds National de la Recherche Scientifique (Belgium). J.P.D. and C.E. are indebted to the Fonds de la Recherche Fondamentale Collective (Belgium) and to the Fonds de Développement Scientifique (Université Catholique de Louvain) for financial support. We thank the European Community for Access to Research Infrastructure Action of the Improving Human Potential Programme to the EMBL Hamburg Outstation, contract number HPRI-CT-1999-00017. We are grateful to the members of the scientific staff at the EMBL outstation for their help during data collection, and to E. De Hoffmann and R. Rozenberg for mass spectrometry. 
Published in : Journal of Molecular Biology (2001), vol. 311, pp. 751-759

Status: Postprint (Author's version)

\section{References}

1. Chae, H. Z., Robinson, K., Poole, L. B., Church, G., Storz, G. \& Rhee, S. G. (1994). Cloning and sequencing of thiol-specific antioxidant from mammalian brain: alkyl hydroperoxide reductase and thiol-specific antioxidant define a large family of antioxidant enzymes. Proc. Natl Acad. Sci. USA, 91, 7017-7021.

2. Seo, M. S., Kang, S. W., Kim, K., Baines, I. C., Lee, T. H. \& Rhee, S. G. (2000). Identification of a new type of mammalian peroxiredoxin that forms an intramolecular disulfide as a reaction intermediate. J. Biol. Chem. 275, 20346-20354.

3. Zhou, Y., Kok, K. H., Chun, A. C. S., Wong, C. M., Wu, H. W. \& Lin, M. C. M. et al. (2000). Mouse peroxiredoxin V is a thioredoxin peroxidase that inhibits p53-induced apoptosis. Biochem. Biophys. Res. Commun. 268, 921-927.

4. Rropotov, A., Sedova, V., Ivanov, V., Sazeeva, N., Tomilin, A. \& Krutilina, R. et al. (1999). A novel human DNA-binding protein with sequence similarity to a subfamily of redox proteins which is able to repress RNA-polymerase-III-driven transcription of the Alu-family retroposons in vitro. Eur. J. Biochem. 260, 336-346.

5. Yamashita, H., Avraham, S., Jiang, S., London, R. \& Van Veldhoven, P. P. et al. (1999). Characterization of human and murine PMP20 peroxisomal proteins that exhibit antioxidant activity in vitro. J. Biol. Chem. 274, 29897-29904.

6. Knoops, B., Clippe, A., Bogard, C., Arsalane, K., Wattiez, R. \& Hermans, C. et al. (1999). Cloning and characterization of AOEB166, a novel mammalian antioxidant enzyme of the peroxiredoxin family. J. Biol. Chem. 274, 30451-30458.

7. Bryk, R., Griffin, P. \& Nathan, C. (2000). Peroxi-nitrite reductase activity of bacterial peroxiredoxins. Nature, 407, $211-215$.

8. Martin, J. F. (1995). Thioredoxin - a fold for all reasons. Structure, 3, 245-250.

9. Choi, H. J., Kang, S. W., Yang, C. H, Rhee, S. G. \& Ryu, S. E. (1998). Crystal structure of a novel human peroxidase enzyme at $2.0 \AA ̊$ resolution. Nature Struct. Biol. 5, 400-406.

10. Hirotsu, S., Abe, Y., Okada, K., Nagahara, N., Hori, H., Nishino, T. S. \& Hakoshima, T. (1999). Crystal structure of a multifunctional 2Cys peroxiredoxin heme-binding protein $23 \mathrm{kDa}$ /proliferation-associated gene product. Proc. Natl Acad. Sci. USA, 96, 1233312338

11. Schröder, E., Littlechild, J. A., Lebedev, A. A., Errington, N., Vagin, A. A. \& Isupov, M. N. (2000). Crystal structure of decameric 2 Cys peroxiredoxin from human erythrocytes at 1.7 Á resolution. Structure, 8, 605-615.

12. Kleywegt, G. J. \& Jones, T. A. (1997). Detecting folding motifs and similarities in protein structures. Methods Enzymol. 277, 525-545.

13. Jones, T. A., Zou, J.-Y., Cowan, S. W. \& Kjeldgaard, M. (1991). Improved methods for building protein models in electron-density maps and the location of errors in these models. Acta Crystallog. sect. A, 47, 110-119.

14. Jin, D.-Y., Chae, H. Z., Rhee, S. G. \& Jeang, K.-T. (1997). Regulatory role for a novel human thioredoxin peroxidase in NF-kappaB activation, J. Biol. Chem. 272, 30952-30961.

15. Sagone, A. L., Decker, M. A., Wells, R. M. \& Democko, C. (1980). A new method for the detection of hydroxyl radical production by phagocytic cells. Biochim. Biophys. Acta, 628, 90-97.

16. Dauter, Z., Dauter, M. \& Rajashankar, K. R. (2000). Novel approach to phasing proteins: derivatization by short cryo-soaking with halides. Acta Crystallog. sect. D, 56, 232-237.

17. Otwinowski, Z. \& Minor, W. (1997). Processing of X-ray diffraction data collected in oscillation mode. Methods Enzymol. 276, 307326.

18. Brünger, A. T. (1992). The free $R$ value: a novel statistical quantity for assessing the accuracy of crystal structures. Nature, $355,472-$ 475 .

19. Sheldrick, G. M., Dauter, Z., Wilson, K. S., Hope, H. \& Sieker, L. C. (1993). The application of direct methods and Patterson interpretation to high-resolution native protein data. Acta Crystallog. sect. D, 49, 18-23.

20. Collaborative Computational Project Number 4 (1994). The CCP4 suite: programs for protein crystallography. Acta Crystallog. sect. D, $50,760-763$.

21. Otwinowski, Z. (1991). Maximum likelihood refinement of heavy atom parameters. In Isomorphous Replacement and Anomalous Scattering (Wolf, W., Evans, P. R. \& Leslie, A. G. W., eds), pp. 80-86, SERC Daresbury Laboratory, UK 
Published in : Journal of Molecular Biology (2001), vol. 311, pp. 751-759

Status: Postprint (Author's version)

22. Cowtan, K. (1994). DM, an automated procedure for phase improvement by density modification. Joint CCP4 ESF-EACBM Newsletter Protein Crystallog. 31, 24-28.

23. Perrakis, A., Morris, R. M. \& Lamzin, V. S. (1999). Automated protein model building combined with iterative structure refinement. Nature Struct. Biol. 6, 458-463.

24. Sheldrick, G. M. \& Schneider, T. R. (1997). SHELXL: high-resolution refinement. Methods Enzymol. 277, $319-343$.

25. Laskowski, R. A., MacArthur, M. W., Moss, D. S. \& Thornton, J. M. (1993). PROCHECK: a program to check the stereochemical quality of protein structures. J. Appl. Crystallog. 26, 283-291.

26. Kraulis, P. J. (1991). MOLSCRIPT: a program to produce both detailed and schematic plots of protein structures, J. Appl. Crystallog. 24, 946-950.

27. Merritt, E. A. \& Bacon, D. J. (1997). Raster3D: photorealistic molecular graphics. Methods Enzymol. 277, $505-524$.

28. Read, R. J. (1986). Improved Fourier coefficients for maps using phases from partial structures with error. Acta Crystallog. sect. A, 42, $140-149$.

29. Nicholls, A., Bharadway, R. \& Honig, B. (1993). GRASP: graphical representation and analysis of surface properties. Biophys. J. 64, 166-170. 